\title{
Editorials
}

\section{Diagnosis of polycythaemia}

It is generally accepted that an adult has some form of polycythaemia if there is a persistently raised haematocrit (men $>0.51$, women $>0.48$ ) in blood samples taken without venous occlusion. Precise diagnosis of the type of polycythaemia is necessary not only from the point of view of prognosis and treatment but also as a stimulus to a better understanding of the aetiology, pathogenesis, molecular, and genetic basis of these disorders.

Red cell mass measurement is fundamental to the diagnostic pathway of polycythaemias. Red cell mass values are best expressed as a percentage variation from that predicted for a normal individual of the same height and weight. Expression as $\mathrm{ml} / \mathrm{kg}$ total body weight is not recommended as it does not consider that fatty tissue is less vascular than lean tissue. A red cell mass $25 \%$ or more above the mean normal value for that patient is deemed raised and constitutes an absolute polycythaemia, while patients with raised packed cell volume (PCV) values, but normal red cell mass, have apparent polycythaemia. ${ }^{1}$ The possibility that an absolute polycythaemia has not been found because iron deficiency is limiting erythropoiesis should be considered. Conversely, patients with splenomegaly may have a raised red cell mass accompanied by a $\mathrm{PCV}$ in the high normal range.

Absolute polycythaemia can be divided into three types: - primary polycythaemia where there is an intrinsic abnormality of the bone marrow

- secondary polycythaemia where the erythrocytosis is a reaction to pathology or physiological influence outside the bone marrow (mediated usually by increased erythropoietin secretion)

- idiopathic erythrocytosis, a heterogenous group comprising patients who do not fit clearly into one of the first two groups.

The diagnostic strategy in patients with an absolute polycythaemia should be rigorous, bearing in mind the various pathogenic mechanisms, and remembering that it is possible for two relevant pathologies to coexist, especially in elderly patients.

Polycythaemia vera (PV, polycythaemia rubra vera), a clonal myeloproliferative disorder resulting from somatic mutation of a haemopoietic stem cell, is the cause of primary polycythaemia in nearly all patients. Recently, the possibility that other types of abnormality within the bone marrow may cause primary polycythaemia has emerged. While more mechanisms will certainly be established in the future, the only defined abnormality at present is an exceedingly rare congenital (polyclonal) and usually familial condition where a point mutation in the erythropoietin receptor gene leads to truncation of the cytoplasmic portion of the erythropoietin receptor. ${ }^{2}$ As this is normally responsible for switching off signal transduction in erythroid cells, the truncation leads to an erythrocytosis.

There are numerous causes of secondary polycythaemia, notably hypoxaemia (defined as an arterial oxygen saturation less than 92\%), renal disease, tissue hypoxia (as in mutant high oxygen affinity haemoglobins detected by measuring p50 on the oxygen dissociation curve), and various tumours such as hepatomas, fibroids, and cerebellar haemangioblastomas, as well as the rare congenital abnormality of autonomous high erythropoietin production.

Central to the diagnostic process in polycythaemia is not only estimation of arterial oxygen saturation (including during sleep if appropriate ${ }^{3}$ ), which is most conveniently measured by pulse oximetry, but also abdominal ultrasound. The latter may demonstrate renal or hepatic disease and will allow an estimate of spleen size. Patients with an absolute polycythaemia and palpable splenomegaly (without other cause) fulfil the criteria for the diagnosis of PV, but in those without a palpable spleen who nevertheless appear to have some splenic enlargement by ultrasound, the likelihood of PV is strengthened ${ }^{4}$ and that of secondary polycythaemia weakened.

While measurement of serum erythropoietin is now both relatively simple and reliable, it has proved less useful than hoped in distinguishing different types of polycythaemia. ${ }^{5}$ Only about half of patients with secondary polycythaemia have raised erythropoietin, and about two thirds of those with PV have a reduced concentration. However, it is one of the newer tests, in addition to spleen sizing and karyotyping, that have helped to modernise the diagnostic criteria of PV. ${ }^{6}$

The clonal nature of PV cell proliferation is well established. The pluripotent nature of the bone marrow cell concerned results in raised neutrophils $\left(>10 \times 10^{9} / 1\right)$ and/or platelets $\left(>400 \times 10^{9} / \mathrm{l}\right)$ as well as red cells in the peripheral blood of a large proportion of patients. Except in the small number of patients (15\%) with PV who have an easily identifiable cytogenetic abnormality ${ }^{7}$ (such as $20 \mathrm{q}^{-}$), clonality is difficult to demonstrate. Currently it depends on research techniques that identify $\mathrm{X}$ chromosome inactivation patterns and therefore applies only to women. ${ }^{8}$ Bone marrow examination is useful not only for karyotyping but also to assess histology that can show characteristic changes in PV. However, standardised objective criteria of histological assessment are not yet established for precise diagnostic use. ${ }^{9}$

Another expression of the abnormal proliferating clone in PV is its behaviour in culture. Evidence that red cell precursors in the peripheral blood will grow in serum containing culture media to form colonies without the addition of further erythropoietin (endogenous erythroid colonies) has been widely assessed as a potentially useful test to distinguish PV from other polycythaemias. ${ }^{10}$ This is not a routine procedure and must be standardised in each laboratory to provide a discriminatory test. Further elucidation of abnormal responses of the PV clone to erythropoietin and other growth factors may permit more reliable tests to be developed as well as perhaps enabling the subdivision of PV into types with differing genetic constitutions.

The term idiopathic erythrocytosis (benign or pure erythrocytosis) is by definition used for patients where, despite adequate investigation, no cause for absolute polycythaemia has been established. This is a heterogenous 
group at present, but with increasing understanding of pathogenesis some will be found to have identifiable congenital or acquired abnormalities of erythropoiesis. ${ }^{11}$ Some individuals with only mildly raised red cell mass probably represent a physiological extreme, while others may be at an early stage of evolution into PV or some clearly recognisable secondary polycythaemia.

Apparent polycythaemia (Geisbock's syndrome, stress, pseudo or relative polycythaemia) is defined as raised PCV and normal red cell mass. The aetiology is imprecise as in idiopathic erythrocytosis. Approximately one third of patients have a reduced plasma volume. Some are probably physiological variants showing a red cell mass near the upper normal limit for that patient's height and weight combined with plasma volume near the lower limit. Others may be evolving into an absolute polycythaemia with causes as previously described. In the remainder, various associations have been observed, particularly with hypertension, obesity, smoking, and arterial hypoxaemia, although the mechanisms are not yet clear. ${ }^{12}$ In about one third of these patients, PCV tends to revert to normal over time. Ideally, individuals with apparent polycythaemia should undergo the rigorous investigation carried out in patients with absolute polycythaemia. In practice, personal and financial considerations generally limit investigation of patients with apparent polycythaemia to a full blood count, biochemistry screen, arterial oxygen saturation, carbon monoxyhaemoglobin concentration, and an abdominal ultrasound.

M MESSINEZY

T C PEARSON

Division of Haematology, United Medical and Dental Schools,

St Thomas's Hospital,

Lambeth Palace Road, London SE1 7EH, UK.

Pearson TC, Guthrie DL, Simpson J, et al. Interpretation of measured red cell mass and plasma volumes in adults. Br f Haematol 1995;89:748-56.

2 de la Chapelle A, Träskelin A-L, Juvonen E. Truncated erythropoietin receptor causes dominantly inherited benign human erythrocytosis. Proc Natl Acad Sci 1993;90:4495-9.

3 Moore-Gillon JC, Treacher DF, Gaminara EJ, et al. Intermittent hypoxia in patients with unexplained polycythaemia. BMF 1986;293:588-90.

4 Messinezy M, Macdonald LM, Nunan TO, et al. Spleen sizing by ultrasound in polycythaemia and thrombocythaemia: comparison with SPECT. Br f Haematol 1997;98:103-7.

5 Birgegård G, Wide L. Serum erythropoietin in the diagnosis of polycythaemia and after phlebotomy treatment. Br f Haematol 1992;81:603-6.

6 Pearson TC, Messinezy M. The diagnostic criteria of polycythaemia rubra vera. Leuk Lymphoma 1996;22(supp1 1):87-93.

7 Diez Martin JL, Graham DL, Petitt RM, et al. Chromosome studies in 104 patients with polycythemia vera. Mayo Clin Proc 1991;66:287-99.

8 Gale RE, Wainscoat JS. Clonal analysis using X-linked DNA polymorphisms. Br F Haematol 1993;85:2-8.

9 Peterson P, Ellis JT. The bone marrow in polycythemia vera. In: Wasserman LR, Berk PD, BerlinNI, eds. Polycythaemia vera and the myeloproliferative disorders. Philadelphia: WB Saunders, 1995:31-53.

10 Reid CDL. The significance of endogenous erythroid colonies (EEC) in haematological disorders. Blood Rev 1987;1:133-40.

11 Messinezy M, Sawyer B, Westwood NB, et al. Idiopathic erythrocytosisadditional new study techniques suggest a heterogenous group. Eur f Haematol 1994:53:163-7.

12 Pearson TC. Apparent polycythaemia. Blood Rev 1991;5:205-13.

\section{Early diagnosis of myocardial infarction: why measure cardiac enzymes?}

A typical district general hospital will see approximately 5000 patients each year in whom a diagnosis of acute coronary syndrome is suspected. This will include not only patients with unstable angina or acute myocardial infarction (AMI) but also patients presenting with chest pain of unknown cause, sudden onset of breathlessness, rhythm disturbance or worsening ischaemic heart disease. Is rapid biochemical diagnosis required?

The decision to admit to hospital and whether the patient will be placed in the coronary care unit (CCU) or a general ward/low dependency facility will depend on the initial clinical assessment plus electrocardiography (ECG). Both of these are known to be imperfect approaches$11.8 \%$ of patients with AMI are sent home from the accident and emergency department ${ }^{1}$ and patients without coronary artery disease inappropriately occupy $25 \%$ of CCU beds. Using postmortem examination as the gold standard, biochemical diagnosis of AMI has been shown to be superior to ECG. ${ }^{2}$ Although the sensitivity of ECG is only $75 \%$ on presentation, ST segment elevation has $99.7 \%$ specificity for AMI, identifying the group that will have maximum benefit from thrombolytic treatment. ${ }^{3}$ ECG criteria can be used to select for thrombolysis patients who present with ST segment elevation or bundle branch block. Giving thrombolytic agents on the basis of clinical features alone will confer no benefit in the absence of ST segment elevation and can result in additional morbidity and mortality in the patient both with and without ischaemic heart disease. For example, dissecting aneurysm can present with chest pain and thrombolysis in such cases has a spectacular effect on outcome. Widespread use of thrombolytic treatment in the absence of ECG data by paramedic staff cannot be justified.

Most cases do not have a clear cut presentation. An audit of cases presenting to the Mayday Hospital (Thornton Heath, Surrey, UK) who had cardiac enzyme analysis showed that (with diagnosis based on WHO criteria for AMI) only $9.6 \%$ of patients presenting with chest pain had ST segment elevation on presentation. On the basis of biochemical testing a further $2.4 \%$ were diagnosed with non-Q wave AMI and $7.8 \%$ had biochemical changes characteristic of myocardial damage. Sixty six per cent of cases had a final diagnosis of stable angina or nonischaemic chest pain.

Clearly the ECG is not a diagnostic but a management tool that should be used to select patients for thrombolytic treatment. This constitutes the minority of patients investigated. Most will require biochemical confirmation or exclusion of AMI. Identification of patients without ECG changes who have either a non-Q wave AMI or unstable angina with minor myocardial damage is important. These patients have a short term mortality that exceeds that of patients with $\mathrm{Q}$ wave $\mathrm{AMI}$, and they require early intervention. The ECG has superior diagnostic sensitivity to all currently available biochemical markers in samples taken on admission. ${ }^{4}$ This is because of the kinetics of release of biochemical markers from ischaemic tissue and the problem of single measurement compared with a population based reference range. Early diagnosis of myocardial infarction can be achieved by serial measurement of myoglobin, ${ }^{5}$ creatine kinase $(\mathrm{CK})^{6}$ or its $\mathrm{MB}$ isoenzyme (CK-MB). ${ }^{7}$ Diagnosis can be complete within 24 hours of presentation. By using a combination of a short time 
window marker plus a long time window marker, measured serially in the same patient, the diagnostic time window can be reduced to as little as four hours from admission. ${ }^{8}$

The strategy in the UK for biochemical diagnosis is usually based around a protocol of a combination of daily measurement of CK, aspartate transaminase (AST), and lactate dehydrogenase (LDH). The diagnostic superiority of CK-MB has been established both by clinical studies ${ }^{9}$ and rigorous statistical comparison with other markers. ${ }^{10}$ It is considered the standard marker in Europe and North America. The technology for rapid, accurate, and precise mass measurement of CK-MB is available in the UK and is the method of choice for confirmation or exclusion of AMI by conventional criteria. However, the cardiac enzyme strategy in most hospitals is both diagnostically inadequate and does not provide information within a useful time frame for medical decision making. Two other factors combine to make the present situation unacceptable.

The first is the development of assays for the cardiac specific marker proteins troponin $\mathrm{T}$ and troponin $\mathrm{I}$. These markers are $100 \%$ sensitive and specific for cardiac damage. They allow prognostic risk stratification for patients with a diagnosis of $\mathrm{AMI}$ and unstable angina. The information cannot be obtained from CK-MB measurement or ECG. ${ }^{11-13}$ The cardiac specific markers troponin $\mathrm{T}$ and troponin I will replace CK-MB as they allow diagnosis to be complete within 12 hours of admission, are more sensitive and specific, and have prognostic value. They will become the gold standard for diagnosis. ${ }^{14}$

The second is the evolution of point of care testing (POCT). Rapid quantitative analysis of $\mathrm{CK}$ can be performed reliably on whole blood using small bench-top analysers. POCT devices are currently available for the detection of myoglobin, CK-MB, troponin T, and troponin I. These devices can be used in the emergency department or the CCU to produce results within 20 minutes from first blood sampling. Although these devices are currently semiqualitative, quantitative analysis of cardiac markers with measurement on whole blood is already undergoing clinical trials.

The rapid provision of an accurate biochemical diagnosis is not required for patients with characteristic ECG changes, but is needed to prevent inappropriate discharge of those with cardiac damage, and to expedite the discharge of those without active ischaemic heart disease. Thus, decision making will initially divide patients into those with and without ST segment elevation. The former require thrombolysis and admission to the CCU. Those without ST segment elevation can be assessed rapidly by biochemical testing to confirm or exclude AMI and for risk stratification. This will require measurement of a rapid marker, such as myoglobin, and a troponin. The speed with which both POCT and laboratory instrumentation can provide accurate diagnosis (within 20 minutes) will mean these data can be used to guide management decisions. The chest pain evaluation unit, ${ }^{15}$ using rapid serial enzyme measurement reduces both the need for unnecessary admissions and the number of patients with AMI incorrectly discharged. In the CCU, rapid enzyme measurement allows early discharge of patients without AMI. ${ }^{6}$ The speed of provision of diagnostic information can influence subsequent management, altering length of stay and hence patient episode cost. ${ }^{6}{ }^{16}$ The current concept of admitting all patients with suspected AMI to the CCU or hospital followed by a wait of two to three days for enzyme analysis cannot be sustained because of the economic cost of inappropriate admissions and the length of hospital stay before diagnosis is confirmed or excluded. The diagnostic superiority of troponin $\mathrm{T}$ and I measurement means that these markers should replace existing strategies. A single troponin measurement 12 hours from admission has $100 \%$ sensitivity and specificity for AMI. Troponins identify at risk patients missed by conventional markers, and troponin measurement can be combined with ECG to stratify patients according to outcome, ${ }^{12} 1317$ hence identifying patients for early discharge or investigation.

The issue is one of cost. The total cost of troponin measurement is $£ 4.50$ to $£ 7.50$, similar to CK-MB mass but twice the price of three CK measurements $(£ 3.30$ in our laboratory). However, as a CCU bed costs $£ 405 /$ day, and a medical bed costs $£ 183 /$ day, any reduction in length of stay will rapidly repay the additional analytical cost, while there is a quality benefit from diagnosis of high risk patients. $^{18}$

The issue of which troponin is better can be debated ${ }^{19}$ with arguments over specificity in renal failure. ${ }^{20} 21$ Troponin $\mathrm{T}$ has been more extensively clinically validated while direct comparison of tropinin $\mathrm{T}$ and I suggests that troponin $\mathrm{T}$ is the more sensitive in the cardiac group, the clinically most significant group of patients. ${ }^{22} 23$

In conclusion, cardiac enzymes are integral to the diagnosis and management of patients in whom acute coronary syndromes are suspected. They are not needed to diagnose the minority, who present with ST segment elevation, but they are essential for the early differential diagnosis of those at highest and lowest risk. These patients can be diagnosed by biochemical tests alone, to expedite management decisions. Cardiac troponin measurement is the most clinically effective test, offering prognostic information to the clinician unavailable by other investigations, and should be part of the modern testing strategy. The role of biochemical testing is as part of a structured decision making protocol providing accurate and timely information that can be used to guide patient management.

If the test results are not used, then biochemical testing becomes an irrelevance. The current antediluvian practice of offering daily measurements of $\mathrm{CK}$, AST, and $\mathrm{LDH}$ serves only to provide a postscript to the discharge summary or death, whichever comes first, and should be abandoned in favour of an integrated approach using outcome related tests (troponin $\mathrm{T}$ or $\mathrm{I}$ ) to alter patient management directly. There are financial benefits in terms of reduction of patient episode cost while accurate diagnosis from modern tests will aid prognostic decision making. Converting this into routine clinical practice is the challenge both for the laboratory and the clinician.

P O COLLINSON

Consultant Chemical Pathologist,

Mayday Hospital,

London Road,

Thornton Heath, Surrey CR7 7YE, UK

email:poctrop@poctrop.demon.co.uk

1 Emerson PA, Russel NJ, Wyatt J, et al. An audit of doctors management of patients with chest pain in the accident and emergency department. Quart patients with chest pain

2 McQueen MJ, Holder D, El-Maraghi N. Assessment of the accuracy of serial electrocardiography in the diagnosis of acute myocardial infarction. serial electrocardiography in

3 Fibrinolytic Therapy Trialists' Collaborative Group. Indications for fibrinolytic therapy in suspected acute myocardial infarction: collaborative overview of early mortality and major morbidity results from all randomised trials of more than 1000 patients. Lancet 1994;343:311-22

4 Bakker AJ, Koelemay MJ, Gorgels JP, et al. Failure of new biochemical markers to exclude acute myocardial infarction at admission. Lancet 1996; 342:1220-2.

5 Lindahl B, Venge P, Wallentin L. Early diagnosis and exclusion of acute myocardial infarction using biochemical monitoring. Coron Artery Dis 1995;6:321-8

6 Collinson PO, Ramhamadamy EM, Stubbs PJ, et al. Rapid diagnosis of patients with acute chest pain reduces patient stay in the coronary care unit. Ann Clin Biochem 1993;30:17-22.

7 Collinson PO, Rosalki SB, Kuwana T, et al. Early diagnosis of acute myocardial infarction by CK-MB mass measurements. Ann Clin Biochem 1992;29: 43-7.

8 Collinson PO, Stubbs PJ. 4 hour "rule-in" diagnosis of acute myocardial infarction for early risk stratification by creatine kinase increment (CK change). Ann Clin Biochem 1996;33:308-13. 
9 Lee TH, Goldman L. Serum enzyme assays in the diagnosis of acute myocardial infarction. Ann Intern Med 1986;105:221-33.

10 Leung FY, Galbraith LV, Jablonsky G, et al. Re-evaluation of the diagnostic utility of serum total creatine kinase and creatine kinase-2 in myocardial infarction. Clin Chem 1989;35:1435-40.

11 Stubbs P, Collinson P, Moseley D, et al. Prospective study of the role of cardiac troponin $\mathrm{T}$ in patients admitted with unstable angina. $B M F$ 1996;313: 262-4.

12 Stubbs PJ, Collinson PO, Moseley D, et al. Prognostic significance of admission troponin T concentrations in patients with myocardial infarction. Circulation 1996;94:1291-7.

13 Antman EM, Tanasijeevic MJ, Thompson B, et al. Cardiac specific troponin I levels to predict the risk of mortality in patients with acute coronary syndromes. N Engl f Med 1996;335:1342-9.

14 Ryan TJ, Anderson JL, Antman EM, et al. ACC/AHA Guidelines for the management of patients with acute myocardial infarction. Circulation 1996; 94:2341-50.

15 Hedges JR, Gibler WB, Young G, et al. Multicenter study of creatine kinase-MB use: effect on chest pain clinical decision making. Acad Emerg kinase-MB use: effect on chest pain clinical decision making. Acad Emerg
Med 1996;3:7-15.

16 Hedges JR. Cardiac enzyme availability and hospital length of stay. Clin Chem 1997;43:249-50.
17 Lindahl B, Andren B, Ohlsson J, et al. Risk stratification in unstable coronary artery disease. Additive value of troponin determinations and pre-discharge stress tests. Eur Heart $\mathcal{F}$ 1997;18:762-70.

18 Collinson PO. Cost economics of new cardiac markers-CK-MB and cardiac troponins. In: Kaski CJ, Holt D, eds. Myocardial damage: early detection by novel biochemical markers. Amsterdam: Kluwer Academic Publishers. [In press.]

19 Collinson PO. To $\mathrm{T}$ or not to $\mathrm{T}$, that is the question. Clin Chem 1997;43:421-3.

20 Haller C, Zehelein J, Remppis A, et al. Cardiac troponin T in patients with renal failure [abstract]. 7 Am Coll Cardiol 1997;29(suppl):234A.

21 McLaurin MD, Apple FS, Voss EM, et al. Cardiac troponin I, cardiac troponin $\mathrm{T}$ and creatine kinase $\mathrm{MB}$ in dialysis patients without ischaemic heart disease Clin Chem 1997;43:976-82.

22 Christensen R, Duh SH, Newby K, et al. Cardiac troponin T and cardiac troponin I: relative values in short term risk stratification of patients with acute coronary syndromes. Clin Chem. [In press.]

23 Lindahl B, Venge PL, Wallentin L. Comparison of troponin T and troponin I for prediction of mortality in unstable coronary artery disease [abstract]. Eur Heart $\mathcal{F}$ 1997;18(suppl): 123. 